\title{
We Présentation Actualité de la clinique institutionnelle et du handicap
}

Claude Wacjman ${ }^{[1]}$

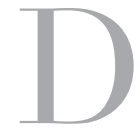

epuis plus de dix ans les établissements recevant des enfants handicapés sont confrontés à deux ordres de faits législatifs qui ont une influence sur l'institution de la nature et de l'organisation des soins. On fait référence à la réorganisation du secteur médico-social par la loi 2002-2 et la prise en compte généralisée du handicap dans la loi 2005. L’application de ces lois annoncées par la loi sur l'autisme comme handicap (1996) a une influence sur les pratiques de soins, notamment celles se réclamant de la psychanalyse. La problématique de ce numéro se centrera sur la possibilité de prodiguer des soins sur le versant psychique, d'organiser le travail institutionnel pour maintenir cette orientation sur les plans individuel et institutionnel, tout en respectant l'orientation législative. Des textes abordant théories, opinions, expériences et pratiques à ce propos, sont présentés dans l'optique d'une diversification des orientations de travail, rendue possible en évitant le morcellement des pratiques individuelles et en promouvant un travail pluridisciplinaire, respectueux et hors de toute querelle, sans renier des positions affirmées. Nous avons voulu situer le travail des professionnels, directeurs, psychiatres, psychologues et éducateurs, ainsi que le point de vue des parents, dans une optique aussi large que possible, pour éviter les aspects destructifs et néfastes pour les personnes handicapées, les patients, les familles et les professionnels, que des querelles irraisonnées ont porté sur le devant de la scène ces derniers temps.

Des sociologues font le point sur la Loi n ${ }^{\circ}$ 2005-102 du 11 février 2005 pour l'égalité des droits et des chances, la participation et la citoyenneté des personnes handicapées, tant au regard des pratiques cliniques et des positions sociétales qu’à celui de l'évolution des pratiques de formation en travail social. Ces aspects sont
[1] Psychologue, Directeur de recherches, Paris Diderot, CRPMS. claude.waciman@ wanadoo.fr 
importants à prendre en compte pour les psychologues travaillant en institution. Cette réflexion se poursuit par l'examen des difficultés des établissements pour jeunes handicapés avec l'Éducation nationale (dont aucun représentant sollicité dans le domaine de la formation n'a pu ou voulu partager ses réflexions avec nous) et par les témoignages d'acteurs du terrain exerçant ou ayant exercé dans des dispositifs institutionnels. La place des parents dans le secteur du handicap a une place importante dans ce numéro, dans l'alliance dans le travail entre parents et établissements, dans des aspects de médiation et par le point de vue exprimé par la plus importante association de parents de personnes handicapées, qui reçoit dans ses établissements affiliés le nombre le plus important de personnes autistes. D'autres auteurs rapportent des séquences de leur travail clinique, éthique et institutionnel.

Une partie historique rappelle au moment du bicentenaire de sa naissance, la carrière d'Edouard Séguin qui, dans le milieu du XIX e siècle instaura la méthode médico-pédagogique dont on constate encore la présence au XXI ${ }^{e}$ siècle. On lit aussi une des premières applications de la psychanalyse dans un état nerveux.

La revue Psychologie Clinique rend hommage au Professeur Roger Misès, dont la carrière a eu des influences exceptionnelles en France et dans d'autres pays sur la clinique institutionnelle au sortir d'une période asilaire, la mise en œuvre des traitements, l'institution des soins psychothérapiques par le travail d'équipe ( $\mathrm{L} a$ cure en institution), la classification dynamique des troubles de l'enfance et de l'adolescence (CFTMEA, notamment l'importance du concept de dysharmonie évolutive), la formation des différents cadres et praticiens du champ du handicap et du champ sanitaire en France durant de longues décennies. 